\title{
Numerical Wave Tank Identification of Nonlinear Discrete Time Hydrodynamic Models
}

\author{
Josh Davidson, Simone Giorgi \& John V. Ringwood \\ Centre for Ocean Energy Research \\ Maynooth University, Ireland
}

\begin{abstract}
Hydrodynamic models are important for the design, simulation and control of wave energy converters (WECs). Linear hydrodynamic models have formed the basis for this and have been well verified and validated over operating conditions for which small amplitude assumptions apply. At larger amplitudes a number of nonlinear effects may appear. One of these effects is due to the changing bouyancy force as the body moves in and out of the water. In this paper we look at identifying a nonlinear static block to be added the linear hydrodynamic model to account for this effect. The parameters for this nonlinear block are identified from WEC experiments simulated in a numerical wave tank (NWT). The parameters for the linear hydrodynamic model are also identified from NWT experiments. Here we explore the use of a discrete time linear hydrodynamic model which is well suited to the identification procedure.
\end{abstract}

\section{INTRODUCTION}

Hydrodynamic models are important for the design, simulation and control of WECs. Linear hydrodynamic models have formed the basis for this and have been well verified and validated over operating conditions for which small amplitude assumptions apply. At larger amplitudes a number of nonlinear effects may appear. Computational fluid dynamics (CFD) can provide a fully nonlinear simulation, however at a great computational expense. We seek to combine the fidelity of the CFD simulations with the usability of a parametric model, by identifying parametric hydrodynamic models from CFD generated data.

This approach has previously been utilised to identify representative linear parametric hydrodynamic models (Davidson et al. 2013) (Armesto et al. 2014). The next step is to identify a nonlinear parametric hydrodynamic model. Bhinder et al. (2011) used CFD outputs to identify a parameter value for an additional nonlinear term, representing the viscous forces, to be added to a linear hydrodynamic model. Including the nonlinearity, due to the time varying wetted body surface, into a hydrodynmamic model, has been investigated by integrating the pressure over the instantaneous wetted surface of the body at each time step, to calculate nonlinear Froude-Krylov forces (Gilloteaux et al. 2008)(Babarit and LaporteWeywada 2009)(Guérinel et al. 2011). Zurkinden et al. (2014) included nonlinearity into their hydrodynamic model, by representing the hydrostatic restoring force with a cubic polynomial, identified from experimental data.
In this paper we investigate including nonlinear effects onto the linear hydrodynamic model by adding a nonlinear static block. We identify the parameters of the linear model and the nonlinear static block from NWT experiments. Additionally we explore the use of a discrete time linear model which is well suited towards the system identification problem.

The paper is laid out as follows; in Section 2 the structure of the proposed linear and nonlinear models are outlined. Section 3 then details the NWT experiments used to produce the system identification data. Section 4 describes the process of identifying the model parameters from the NWT generated data. An illustrative example demonstrating these methods is then given in Section 5 and the results of the different models compared.

\section{MODEL STRUCTURES}

The structure of the models is based on the superposition of input forces to the body. This is depicted in Figure 1. For the case of a WEC we consider three external forces; the excitation force from the wave, $f_{E}(t)$, the force from the power-take off (PTO), $f_{P T O}(t)$, and the force from the mooring, $f_{M}(t)$. The sum of these three forces is considered the input to the body, $f_{I N}(t)$. We then seek a model that predicts the body's motion, $y(t)$, in response to this input force.

A model structure which has strong appeal is a linear model, which is described in Section 2.1. However, for many operating conditions there may be nonlinear dynamics which can't be captured by the linear 


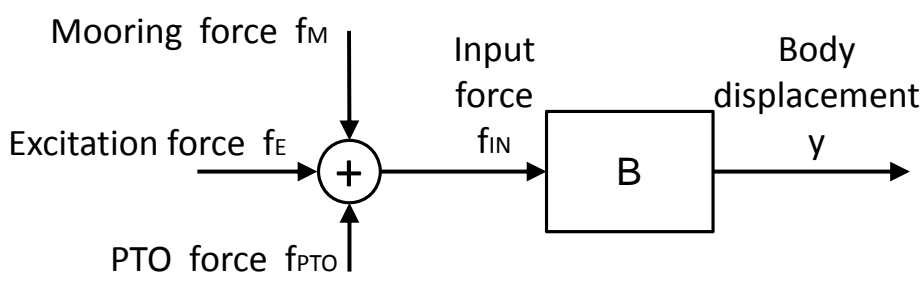

Figure 1: Model structure accounting for excitation, PTO and mooring forces.

model. We then seek to extend the region the linear model description is valid through the addition of a static nonlinear block, described in Section 2.2.

\subsection{Linear hydrodynamic models}

A description and comparison of the different hydrodynamic modelling methods for the dynamic response of marine structures can be found in (Taghipour et al. 2008). At the heart of these modelling methods is the Cummins equation (Cummins 1962), which considers the total force from the fluid being decomposed into three independent pieces; the excitation force, $f_{E}(t)$, the radiation force. $f_{R A}(t)$, and the restoring force, $f_{R E}(t)$. Following this approach, the model structure in Figure 1 also considers the decomposition of the excitation force from the total fluid force.

\subsubsection{Cummins equation}

Cummins' equation is based on Newton's second law of motion, that the product of the mass, $M$, and acceleration, $\ddot{y}(t)$, is equal to the sum of the forces:

$M \ddot{y}(t)=f_{R A}(t)+f_{R E}(t)+f_{I N}(t)$.

By considering the hydrodynamic radiation of a body, with zero forward speed, in an ideal fluid, (Cummins 1962) showed that the radiation forces can be expressed as:

$f_{R A}(t)=-m_{\infty} \ddot{y}(t)-\int_{-\infty}^{t} h_{R A}(t-\tau) \dot{y}(\tau) d \tau$

where the infinite frequency added mass, $m_{\infty}$, and the reduced radiation impedence impulse response function, $h_{R A}(t)$, depend on the body geometry.

The restoring force is due to the mismatch between the gravity and bouyancy forces when the body moves away from equilibrium. For linear hydrodynamic models the restoring force is represented as a linear spring, $f_{R E}(t)=-K y(t)$.

This leads to the Cummins equation:

$$
\left(M+m_{\infty}\right) \ddot{y}(t)+\int_{-\infty}^{t} h_{R A}(t-\tau) \dot{y}(\tau) d \tau+K y(t)=f_{I N}(t)
$$

Traditionally the parameter values for the Cummins equation have been identified using the boundary element method (BEM) and are subject to the linearising assumptions of that method. Identifying the parameters from nonlinear NWT experiments has been shown to develop representative linear models (Davidson et al. 2013) (Armesto et al. 2014), however the parameter identification turns out to be a nonconvex optimisation problem with a high computational requirement and no guarantee of obtaining a global minimum.

An alternative approach explored in this paper is to abandon the Cummins equation and adopt a structure more suited to the system identification problem. System identification involves identifying the parameters of a model, from measured data of the system response to different excitation signals. Owing to the discrete time nature of sampled data measured from experiments, the majority of system identification techniques are based on discrete time models (Ljung 1999). A simple linear discrete time model, the autoregressive model with exogenous input (ARX), is investigated in this paper.

\subsubsection{ARX model}

An ARX model is a very well known type of model for representing a system's next output based on a linear combination of its past outputs and inputs. The ARX model for the system represented by the block $B$, in Figure 1, with an input, $f_{I N}(t)$, and output, $y(t)$, is:

$y(t)+\sum_{i=1}^{n_{a}} a_{i} y(t-i)=\sum_{i=1}^{n_{b}} b_{i} f_{I N}(t-i)$

Unlike the Cummin's equation, which is derived under consideration of physical laws, the ARX model is a black box. A black box model simply reproduces the experimental output data, given the same stimulus, but the internal model structure bears no resemblance to the physical world.

\subsection{Nonlinear models}

A way to introduce a nonlinearity in the relationship between the input $f_{I N}(t)$ and the output $y(t)$ of the model, is utilising a nonlinear static block. Here we look at introducing this static block in two ways; via the well known Hammerstein structure, as detailed in Section 2.2.1, and by using a feedback block oriented model, Section 2.2.2.

\subsubsection{Hammerstein model}

The Hammerstein model consists of the cascade connection of a nonlinear static block followed by a linear dynamic block (see Figure 2). The equation that describes the input-output relationship for a Hammerstein model is (Pearson \& Pottmann 2000):

$y(t)+\sum_{i=1}^{n_{a}} a_{i} y(t-i)=\sum_{i=1}^{n_{b}} b_{i} r\left(f_{I N}(t-i)\right)$,

where, $r()$ is a nonlinear static function. 


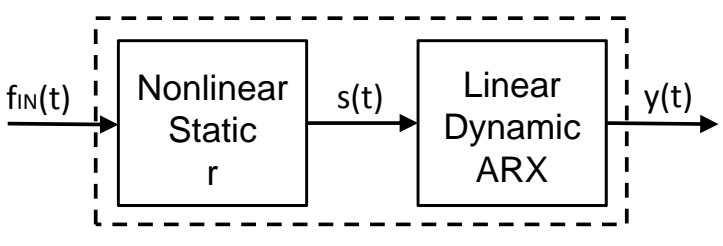

Figure 2: Block diagram of the Hammerstein model.

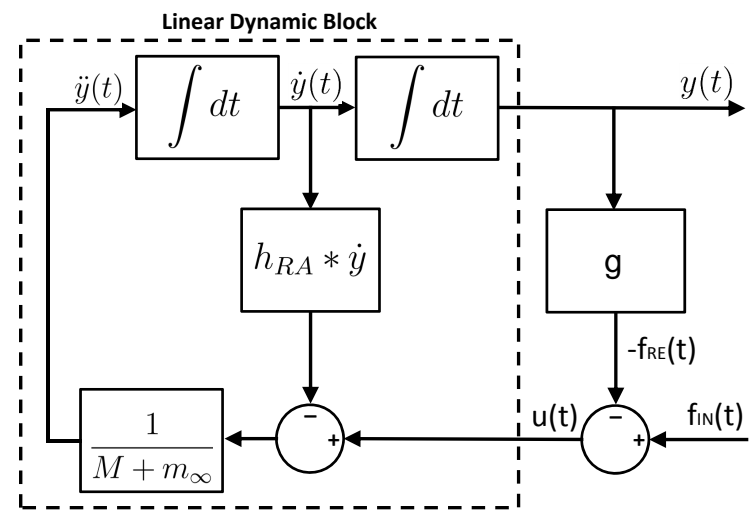

Figure 3: Block diagram representation of Cummins equation

In the Hammerstein model, the static relationship between input and output is given by the product of the static function, $r()$, and the steady-state gain of the ARX model, $D C_{\text {gain }}$. Therefore the characterization of the two blocks is not unique, any pair $\left(D C_{\text {gain }} / \alpha, \alpha r\right)$ will produce the same input and output (Bai 2003). To remove this ambiguity the $D C_{\text {gain }}$ is set equal to one, which allows the steady-state gain for the entire Hammerstein model to be solely represented by the nonlinear static function. In this case, $r\left(f_{I N}(t)\right)$ will represent the static relationship between input force and output body position. The physical interpretation of $r\left(f_{I N}(t)\right)$, is therefore the inverse of the restoring force, $f_{R E}(t)$, in the Cummins equation, which is the static relationship between input body position and output restoring force. The nonlinear static function, $r()$, can therefore be identified separately from the linear dynamic block from knowledge of the restoring force as a function of body position. In this way the Hammerstein model black-box structure is given a shade of 'grey' by considering of the physical meaning of the nonlinear static function.

\subsubsection{Feedback block-oriented model}

The Cummins equation (Equation 3) can be graphically represented by Figure 3, and then further compacted into Figure 4 that represents a Lur'e model structure, which is characterised by a negative feedback (Pearson \& Pottmann 2000). In this negative feedback configuration, the nonlinear static block, $g(y(t))$, relates the body position to the negative of the restoring force $\left(-f_{R E}(t)\right)$. The nonlinear static function, $g()$, can therefore be identified separately from the linear dynamic block from knowledge of the restoring force as a function of body position.

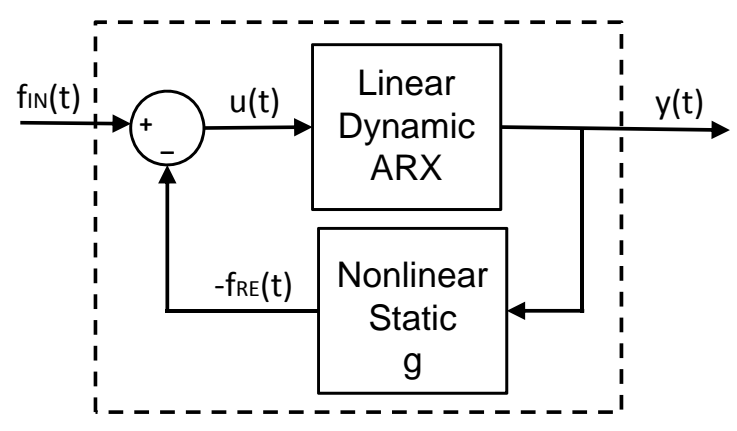

Figure 4: Block diagram of the feedback block-oriented model

\section{NUMERICAL WAVE TANK}

A NWT is a generic name of numerical simulators for nonlinear free surface waves, hydrodynamic forces and floating body motions (Tanizawa 2000). The NWT in this paper uses CFD as the basis for its numerical simulations.

\section{$3.1 \quad C F D$}

The dynamics of fluids is governed by the transfer of mass, momentum and heat. These three processes are described by the Navier-Stokes equations, a set of partial differential equations derived in the early nineteenth century, which form the basis for the analysis of fluid dynamics. In general, these equations have no known analytical solution, however, they may be treated numerically to obtain a solution using CFD.

To solve the Navier-Stokes equations, the continuous partial differential equations are discretised into a system of linear algebraic equations, which can then be solved by computer. That is, the continuum is broken up into finite temporal and spatial portions to transform a calculus problem into an algebraic problem. This is implemented spatially via the mesh with different methods used to discretise the spatial volumes such as: finite volume, finite difference and finite element. The problem is then also discretised temporally using timesteps.

CFD treats the fluid-structure interaction problem, using the scheme outlined in Figure 5. First, the Navier-Stokes equations are solved for the fluid pressure and velocity throughout the domain. The fluid pressure is then integrated over the body's surface, to give the hydrodynamic force on the body. The resulting body motion, due to the hydrodynamic force, is calculated using Newton's laws. The body and the fluid are iterated forward to the next time step and the process is repeated. This is a highly computationally costly way to simulate hydrodynamics. The parametric models described in Section 2, could perform a simulation orders of magnitude faster. The goal therefore, is to only use the computationally expensive CFD simulations once, for a series of NWT experiments to provide information to identify the parameters of Section 2's models. These parametric models can then be used for device simulation. 


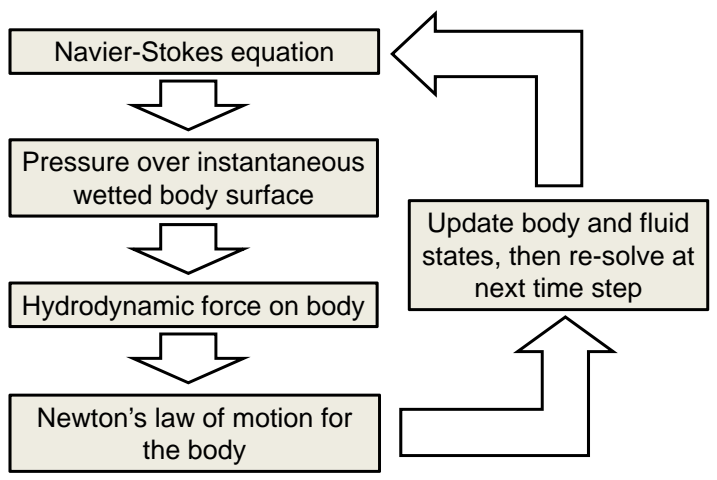

Figure 5: Schematic of CFD process for fluid-structure interaction

\subsection{NWT System Identification Experiments}

The models in Section 2 contain two different types of blocks: the linear dynamic block and the nonlinear static block. This section details the two different NWT experiments, used to identify these two different types of blocks. The NWT Dynamic Experiment is used to identify the linear dynamic block and the nonlinear static block is identified from the NWT Restoring Force Experiment.

NWT Dynamic Experiment: The dynamic experiment involves exciting the body with an input force and measuring the resulting motion. In particular, the PTO force is used to excite the body $\left(f_{I N}(t)=\right.$ $f_{P T O}(t)$ ), because it offers the flexibility of providing any desired signal. Two vectors, containing the sampled input PTO force, $\left\{f_{I N}(t)\right\}$, and the resulting body displacement, $\left\{y_{d}(t)\right\}$, are produced by this experiment for the model identification.

NWT Restoring Force Experiment: This experiment is used to identify the static relationship between the body displacement and the hydrostatic restoring force. The body is displaced through its full range of motion and the resulting hydrostatic force on the body is measured. Two vectors, containing the sampled body displacement, $\left\{y_{r}(t)\right\}$, and the hydrostatic restoring force, $\left\{f_{R E}(t)\right\}$, are produced by this experiment for the model identification.

\subsection{OpenFOAM}

The NWT is implemented using the opensource CFD software OpenFOAM. OpenFOAM has been used to simulate coastal processes (Jacobsen et al. 2012) (Higuera et al. 2013), WEC operation (Palm et al. 2013) (Schmitt et al. 2012), (Iturrioz et al. 2013) and extreme events (Vyzikas et al. 2013). It has been used in NWT applications for hydrodynamic parameter identication (Bonfiglio et al. 2011) (Armesto et al. 2014). Numerous reports and theses are available on the development of a NWT in OpenFOAM (Afshar 2010), (Lambert 2012) and (Cathelain 2013).

The present NWT uses the waveFOAM solver, which was created by Jacobsen et al. (2012) to simulate coastal processes. waveFOAM is an extension of the interFOAM solver, that allows the creation and absorption of waves and currents.

The body motion is simulated by coupling the sixDoFRigidBodyDisplacement solver with waveFOAM's dynamic mesh solver, waveDyMFOAM. Inside the sixDoFRigidBodyDisplacement solver are two useful features: the constraints and restraints functions. The constraints function allows the body to be constrained to only move in designated degrees of freedom. The restraints function allows spring and damper forces to be applied to the body. Palm et al. (2013) modified the restraints function, to couple the solver with a model for mooring forces on a floating body. Here, we follow a similar approach and make modifications to the restraints function to apply a PTO force to the body.

The forces function calculates the hydrodynamic force on the body, by integrating the fluid pressure over the body's surface. The sixDoFRigidBodyDisplacement solver then sums this hydrodynamic force with any user defined constraints and/or restraints forces, and calculates the resulting body motion. The NWT Restoring Force Experiment uses the forces function to calculate the hydrostatic restoring force.

\subsubsection{Mesh}

The mesh is a very important part of the calculation. Poor meshes lead to poor results. There exists a tradeoff between number of cells and computation time. The present NWT is meshed using blockMesh and consists of a central block with a high density of cubic mesh cells. The snappyHexMesh utility is then used to import any body geometry into this central region by removing and refining the mesh cells. snappyHexMesh also allows the mesh around the body to be refined to ensure small enough cell sizes to captures relevant viscous effects and that guidelines such as y-plus values at boundary layers are obeyed.

The domain is split vertically into three regions: water, interface and air. The interface region spans the area where the free surface may appear during the simulation and is set with a high mesh density of uniform vertical cell length. The air region has a low cell density and the length of the cells increase with distance from the interface upwards to the atmosphere boundary. The water region has moderate mesh density and the length of its cells are gradually increased from the interface down to the tank floor. Horizontally the mesh stretches away from the central block, matching the high density in the central region and then growing larger with distance towards the tank wall to reduce the overall amount of cells needed.

\section{MODEL IDENTIFICATION}

This section outlines the parameter identification procedure for the models discussed in Section 2. It is important to note, that both the ARX block and the nonlinear static block, are linear with respect to the parameters. Therefore, the parameters for these mod- 
els can be identified using linear regression and least squares.

\subsection{Linear regression and least squares}

Let's consider a model with an output, $\hat{z}$, that is a linear combination of $p$ independent variables, $x_{i}$ :

$\hat{z}=\theta_{1} x_{1}+\cdots+\theta_{p} x_{p}$

where the coefficients, $\theta_{i}$, are the unknown parameters (Nelles 2001).

It will be assumed that, $i=1 . . N$ data samples, $\{z(i)\}$, have been measured. The error at each sample is $\varepsilon(i)=z(i)-\hat{z}(i)$. In matrix form:

$\boldsymbol{\varepsilon}=\mathbf{z}-\mathbf{z}=\mathbf{z}-\mathbf{X} \boldsymbol{\theta}$

where:

$\varepsilon=\left[\begin{array}{llll}\varepsilon(1) & \varepsilon(2) & \ldots & \varepsilon(N)\end{array}\right]^{T}$,

$\mathbf{z}=\left[\begin{array}{llll}z(1) & z(2) & \ldots & z(N)\end{array}\right]^{T}$,

$\hat{\mathbf{z}}=\left[\begin{array}{llll}\hat{z}(1) & \hat{z}(2) & \ldots & \hat{z}(N)\end{array}\right]^{T}$,

$\mathbf{X}=\left[\begin{array}{cccc}x_{1}(1) & x_{2}(1) & \ldots & x_{p}(1) \\ x_{1}(2) & x_{2}(2) & \ldots & x_{p}(2) \\ \vdots & \vdots & \ddots & \vdots \\ x_{1}(N) & x_{2}(N) & \ldots & x_{p}(N)\end{array}\right]$

$\boldsymbol{\theta}=\left[\begin{array}{llll}\theta_{1} & \theta_{2} & \ldots & \theta_{p}\end{array}\right]^{T}$.

It's well known that the estimated parameters that minimize the least squares error are:

$\hat{\boldsymbol{\theta}}=\underset{\theta}{\operatorname{argmin}}\left(\boldsymbol{\varepsilon}^{T} \boldsymbol{\varepsilon}\right)=\left(\mathbf{X}^{\mathrm{T}} \mathbf{X}\right)^{-1} \mathbf{X}^{\mathrm{T}} \mathbf{z}$.

If the parameters have also to satisfy the linear equality constraints $\mathbf{A} \boldsymbol{\theta}=\mathbf{d}$, the estimated parameters are:

$\hat{\boldsymbol{\theta}}_{c}=\hat{\boldsymbol{\theta}}-\mathbf{H}^{-1} \mathbf{A}^{T}\left(\mathbf{A} \mathbf{H}^{-1} \mathbf{A}^{T}\right)^{-1}(\mathbf{A} \hat{\boldsymbol{\theta}}-\mathbf{d})$,

where $\mathbf{H}=\mathbf{X}^{T} \mathbf{X}$, and $\hat{\boldsymbol{\theta}}$ is calculated via Equation 13 (Nelles 2001).

\subsection{ARX model identification}

Performing the NWT Dynamic Experiment (see Section 3.2), the signals $\left\{f_{I N}(t)\right\}$ and $\left\{y_{d}(t)\right\}$ for $i=$ $1 \ldots N$, are generated, and they can be utilised as input and output for the identification of the ARX model. Because the system that we are trying to replicate with our model is causal, we impose $n_{a}>n_{b}$ for the ARX. Therefore, the first $n_{a}$ values of the output data are utilised as initial conditions, and the first possible predicted output is for $t=n_{a}+1$. In this case Equations 9, 11 and 12 become:

$\mathbf{z}=\left[\begin{array}{llll}y_{d}\left(n_{a}+1\right) & y_{d}\left(n_{a}+2\right) & \ldots & y_{d}(N)\end{array}\right]^{T}$,

$\mathbf{X}=\left[\begin{array}{cccccc}y_{d}\left(n_{a}\right) & \ldots & y_{d}(1) & f_{I N}\left(n_{a}\right) & \ldots & f_{I N}\left(n_{a}-n_{b}+1\right) \\ y_{d}\left(n_{a}+1\right) & \ldots & y_{d}(2) & f_{I N}\left(n_{a}+1\right) & \ldots & f_{I N}\left(n_{a}-n_{b}+2\right) \\ \vdots & \ddots & \vdots & \vdots & \ddots & \vdots \\ y_{d}(N-1) & \ldots & y_{d}\left(N-n_{a}\right) & f_{I N}(N-1) & \ldots & f_{I N}\left(N-n_{b}\right)\end{array}\right]$

$\hat{\theta}_{\mathbf{a r x}}=\left[\begin{array}{llllllll}a_{1} & a_{2} & \ldots & a_{n_{a}} & b_{1} & b_{2} & \ldots & b_{n_{b}}\end{array}\right]^{T}$.

The estimated parameters are given from Equation 13.

\subsection{Hammerstein model identification}

The first step is to identify the nonlinear static block. The nonlinear static function can be approximated with a linear combination of basis functions, in this way it is possible to apply the linear regression for the identification. For simplicity, the selected basis functions in this paper are polynomials $\left\{x^{0}, x^{1}, x^{2}, \ldots, x^{n_{c}}\right\}$. Therefore, the relationship between the input and the output of the nonlinear static block is:

$\hat{s}(t)=c_{1} f_{I N}(t)+c_{2} f_{I N}^{2}(t)+\ldots+c_{n_{c}} f_{I N}^{n_{c}}(t)$

where, $c_{0}=0$, is imposed (applying a null force the displacement has to be zero). In static conditions, $s(t)=y(t)$ and $f_{I N}(t)=-f_{R E}(t)$, therefore Equation 18 becomes:

$\hat{y}(t)=\sum_{k=1}^{n_{c}} c_{k}\left(-f_{R E}(t)\right)^{k}$

The NWT Restoring Force Experiment (see Section 3.2) generates the signals $\left\{f_{R E}(t)\right\}$ and $\left\{y_{r}(t)\right\}$, that can be utilised as input and output for the identification of the nonlinear static block. In this case, Equation 6 becomes Equation 19 and Equations 9, 11 and 12 become:

$$
\begin{aligned}
& \mathbf{z}=\left[\begin{array}{llll}
y_{r}(1) & y_{r}(2) & \ldots & y_{r}(N)
\end{array}\right]^{T},
\end{aligned}
$$

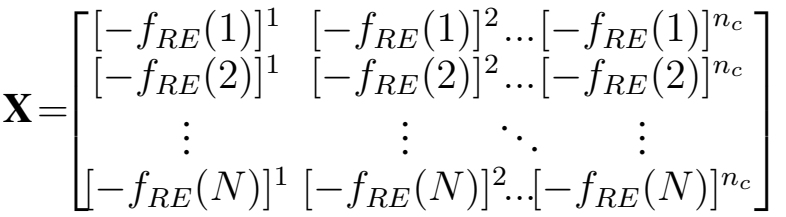

$\hat{\theta}_{\mathbf{H c}}=\left[\begin{array}{llll}c_{1} & c_{2} & \ldots & c_{n_{c}}\end{array}\right]^{T}$.

The estimated parameters are given from Equation 13.

In the second step, the ARX block is identified utilising the signals $\left\{f_{I N}(t)\right\}$ and $\left\{y_{d}(t)\right\}$, generated with the NWT Dynamic Experiment. Now that $\theta_{\mathbf{H c}}$ is known, it is possible to calculate the output $\{s(t)\}$ of the nonlinear static block using Equation 18. In this way, both the input and the output of the ARX is known, and it is therefore possible to identify the ARX parameters $\theta_{\text {Harx }}$, under the constraint that the DC gain of the ARX is equal to one: $D C_{\text {gain }}=\left(\sum_{i=1}^{n_{b}} b_{i}\right) /\left(1+\sum_{i=1}^{n_{a}} a_{i}\right)=1$, that corresponds to an equality constraints on the parameters $\mathbf{A}=[-1,-1, \ldots,-1,1,1, \ldots 1]$ and $d=1$. In this case Equations 9, 11 and 12 become:

$\mathbf{z}=\left[\begin{array}{llll}y_{d}\left(n_{a}+1\right) & y_{d}\left(n_{a}+2\right) & \ldots & y_{d}(N)\end{array}\right]^{T}$

$\mathbf{X}=\left[\begin{array}{cccccc}y_{d}\left(n_{a}\right) & \ldots & y_{d}(1) & s\left(n_{a}\right) & \ldots & s\left(n_{a}-n_{b}\right) \\ y_{d}\left(n_{a}+1\right) & \ldots & y_{d}(2) & s\left(n_{a}+1\right) & \ldots & s\left(n_{a}-n_{b}+1\right) \\ \vdots & \ddots & \vdots & \vdots & \ddots & \vdots \\ y_{d}(N-1) & \ldots & y_{d}\left(N-n_{a}\right) & s(N-1) & \ldots & s\left(N-n_{b}\right)\end{array}\right]$

$\hat{\theta}_{\text {Harx }}=\left[\begin{array}{llllllll}a_{1} & a_{2} & \ldots & a_{n_{a}} & b_{1} & b_{2} & \ldots & b_{n_{b}}\end{array}\right]^{T}$

The estimated parameters are given from Equation 14. 


\subsection{Feedback block-oriented model identification}

The first step is to identify the nonlinear static block. The nonlinear static function, $g()$ can be identified following the same procedure as for the Hammerstein model, utilising the NWT Restoring Force Experiment data $\left\{f_{R E}(t)\right\}$ and $\left\{y_{r}(t)\right\}$. Here the nonlinear static block takes the body displacement as input and outputs the negative of the restoring force: $-f_{R E}(t)=g(y(t))$. Therefore Equations 9, 11 and 12 become:

$\mathbf{z}=\left[\begin{array}{llll}-f_{R E}(1) & -f_{R E}(2) & \ldots & -f_{R E}(N)\end{array}\right]^{T}$,

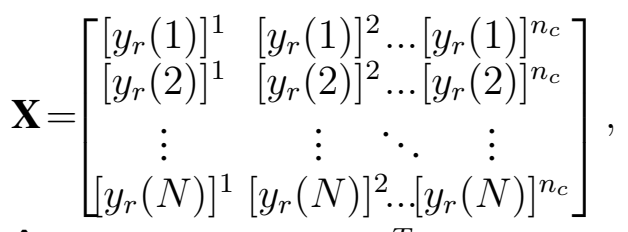

$\hat{\theta}_{\mathbf{F c}}=\left[\begin{array}{llll}c_{1} & c_{2} & \ldots & c_{n_{c}}\end{array}\right]^{T}$.

The estimated parameters are given from Equation 13.

In the second step, the ARX block is identified utilising the signals, $\left\{f_{I N}(t)\right\}$ and $\left\{y_{d}(t)\right\}$, from the NWT Dynamic Experiment. Now that $\theta_{\mathbf{F c}}$ is known, it is possible to calculate the output of the nonlinear static block, $\left\{g\left(y_{d}(t)\right)\right\}$, and then the input of the forward block: $\{u(t)\}=\left\{f_{I N}(t)\right\}-\left\{g\left(y_{d}(t)\right)\right\}$. In this way both input, $\{u(t)\}$, and output, $\left\{y_{d}(t)\right\}$, of the ARX are known, and it is possible to identify the ARX parameters, $\theta_{\text {Farx }}$, with the procedure explained in Section 4.2.

\section{AN ILLUSTRATIVE EXAMPLE}

In this section we implement the methods described in Sections 2 - 4, to model the heave motion of a test device. The geometry of the test device is a vertical cone, which has strong known nonlinearities in its restoring force, and is thus chosen to illustrate the different model types' capabilities of handling this effect.

The cone has a diameter and height of $1 \mathrm{~m}$ and is orientated with its axis aligned vertically, with the base above the tip. The cone's mass density is $50 \%$ of water, resulting in a submerged draft of $0.8 \mathrm{~m}$. The motion will be constrained to heave only.

In this illustrative example we consider the case where the body is subjected to a relatively low frequency $(0.125 \mathrm{~Hz})$ sinusoidal input PTO force. The frequency of this experiment was intentionally chosen low, to emphasize the static nonlinearity of the restoring force, by reducing other possible velocity dependent nonlinearities. Additionally to focus on the contribution of the nonlinear restoring force, a laminar CFD simulation is run in this experiment, to eliminate any possible nonlinear effects from turbulence. The body is also initially displaced $30 \mathrm{~cm}$ above its equilibrium, allowing its free decay oscillation to be superimposed with the response to the PTO force.

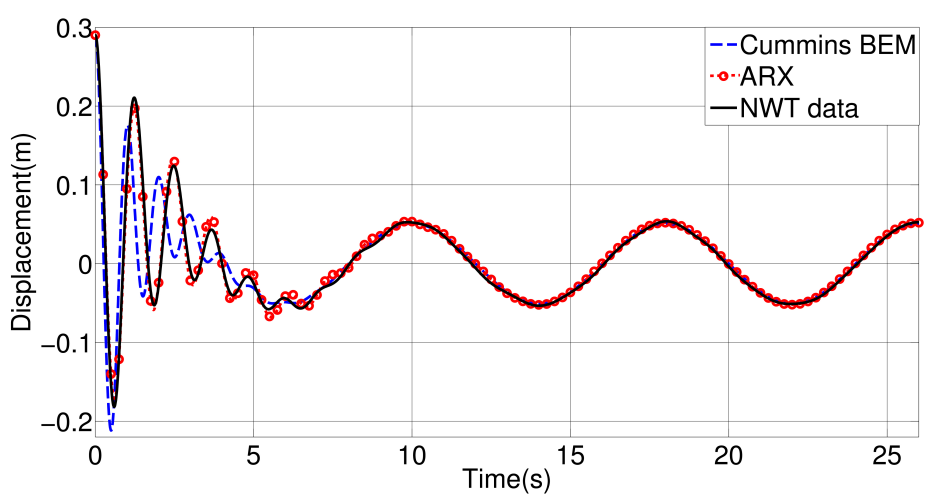

Figure 6: Heave motion predictions by the NWT simulation and two linear hydrodynamic models. The input is a sine of amplitude $240 \mathrm{~N}$ and frequency $0.125 \mathrm{~Hz}$.

The results for the linear ARX model are shown in Section 5.1, and the results from the nonlinear Hammerstein and feedback-block orientated models are shown in Section 5.2.

\subsection{ARX model}

This section compares the results of the linear ARX model against the NWT simulation and also against a linear hydrodynamic model based on the Cummins equations, the Cummins BEM model. The Cummins BEM model's radiation parameter values are identified using the toolbox by Perez \& Fossen (2009), taking as input frequency domain hydrodynamic co-efficients, calculated using the BEM software WAMIT. Its linear restoring force parameter, $K$, is identified as the product of the water density, $\rho$, the gravitational constant, $g$, and the cone's free surface cross-sectional area at equilibrium, $S$, i.e. $K=\rho g S$.

On Figure 6, are heave motion predictions by the NWT simulation and the two linear hydrodynamic models, when the sinusoidal input PTO force has a relatively small amplitude $(240 \mathrm{~N})$. The output contains two parts, the transient due to the initial condition and the steady state response to the harmonic input. It can be seen that, although the three predictions handle the transient part at the start slightly differently, they converge to the same result for the response to the input force. For these small amplitude conditions the linear models are shown here to work well.

A second simulation is then performed of the exact same experiment, except the amplitude of the PTO is increased by a factor of four, to $960 \mathrm{~N}$ (Figure 7). Here we can see that the resulting body motions are much larger and that the performance of the three models diverge. The fully nonlinear NWT simulation exhibits an assymmetrical output, with respect to the equilibrium position, in response to the sinusoidal input, owing to the fact that the cone's geometry makes it harder to push into the water than out. It is not possible for the linear models to replicate this nonlinear behaviour. 


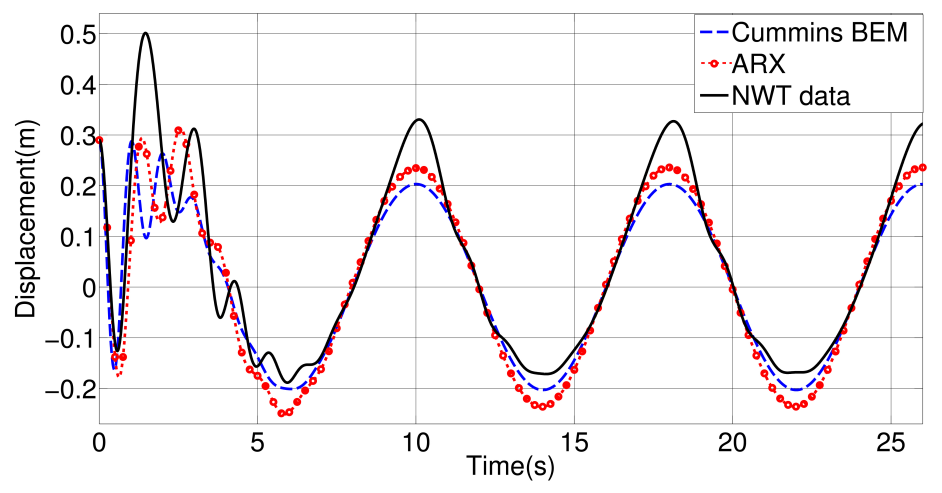

Figure 7: Same scenario as Figure 6 but with a four time larger PTO force applied (960N).
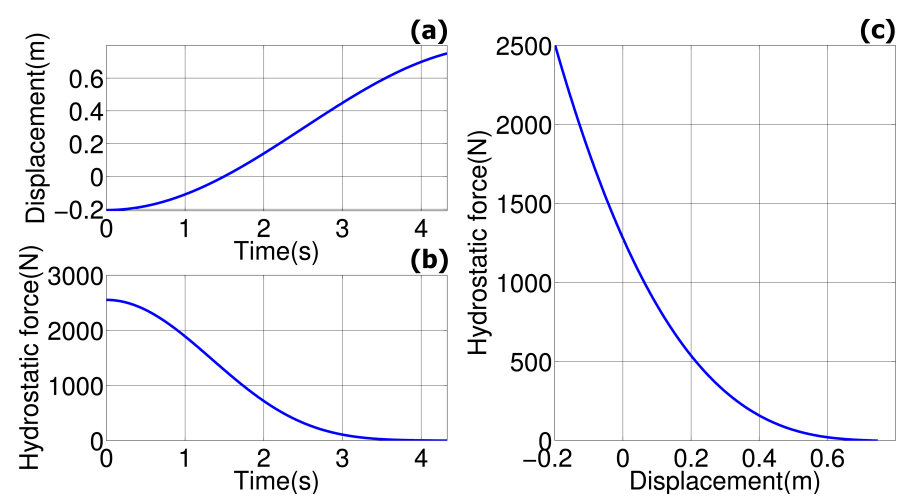

Figure 8: Results from the NWT Restoring Force Experiment

\subsection{The nonlinear models}

The linear models performed well compared to the NWT simulation for the small amplitude conditions, but were unable to handle to larger amplitude conditions in Figure 7. Here we examine the nonlinear models' ability to handle these conditions. First the result of the NWT Restoring Force Experiment and the identification of the nonlinear static blocks is presented, and then the nonlinear models performance for the large amplitude conditions is shown.

The results from the NWT Restoring Force Experiment are shown in Figure 8. Figure 8-(a) shows the motion of the body, starting from the fully submerged position and slowly moving out of the water. Figure 8-(b) shows the hydrostatic force on the body during this motion. Figure 8-(c) then plots the hydrostatic force as a function of position, showing the nonlinear nature of this relationship.

The hydrostatic force versus displacement relationship, obtained in Figure 8-(c), is then transformed to give $r\left(f_{I N}(t)\right)$ for the Hammerstein model and $g(y(t))$ for the feedback block oriented model, shown in Figure 9. Also in Figure 9 is the nonlinear static functions' fit to the NWT data. The order for the polynomial is chosen as the smallest value which leads to a mean squared error (MSE) of less than 3\%, that in our opinion is a good compromise between parsimony and accuracy. From Figure 10, a 7th order polynomial is selected.

The results of the nonlinear models compared to the NWT simulation is shown in Figure 11. Here it can be seen that the nonlinear models outperform their lin- (a)
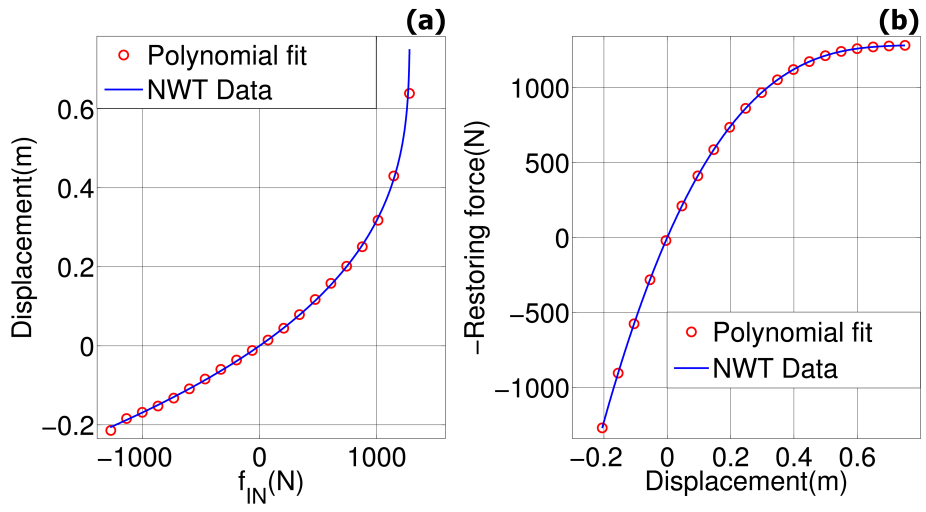

Figure 9: (a) Nonlinear static function $\left.r\left(f_{I N}(t)\right)\right)$ of the Hammerstein model. (b) Nonlinear static function $g(y(t))$ of the feedback model.

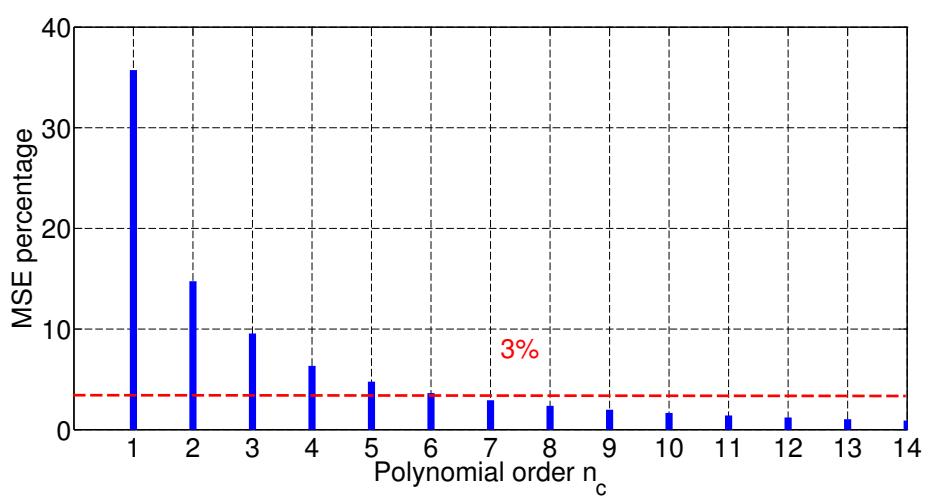

Figure 10: The fitting error for varying polynomial order

ear counterparts' performance for the same case in Figure 7. The nonlinear models are able to replicate the assymetrical steady state response, and better able to reproduce the transient oscillations, particularly the Feedback block-oriented model which does very well.

A further comparison between the linear ARX model and the two nonlinear models is displayed in Figure 12, which plots the fit percentage between the NWT data and the models, for varying model order $n_{a}$ and $n_{b}$. Figure 12 shows the Feedback blockoriented model is best able to fit the NWT data, the Hammerstein was second best and the ARX has the poorest performance.

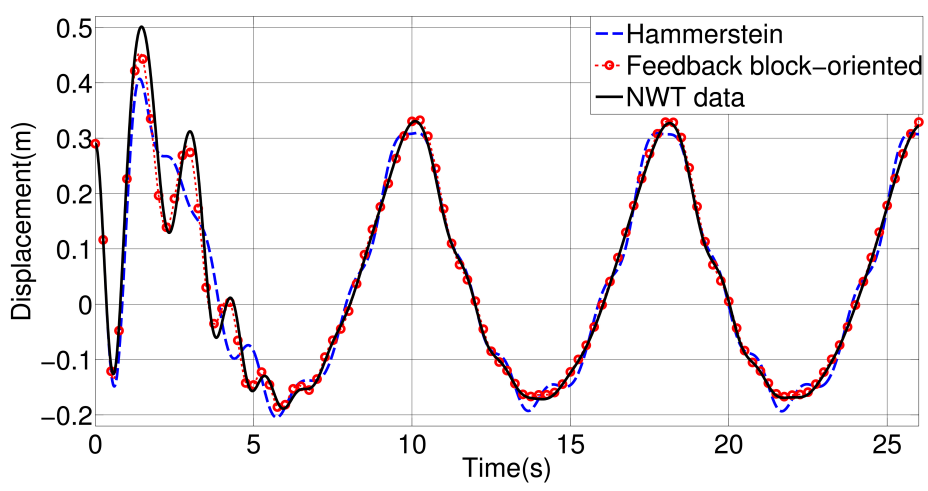

Figure 11: Heave motion predictions by the NWT simulation and the two nonlinear hydrodynamic models for the case with the large amplitude PTO force 


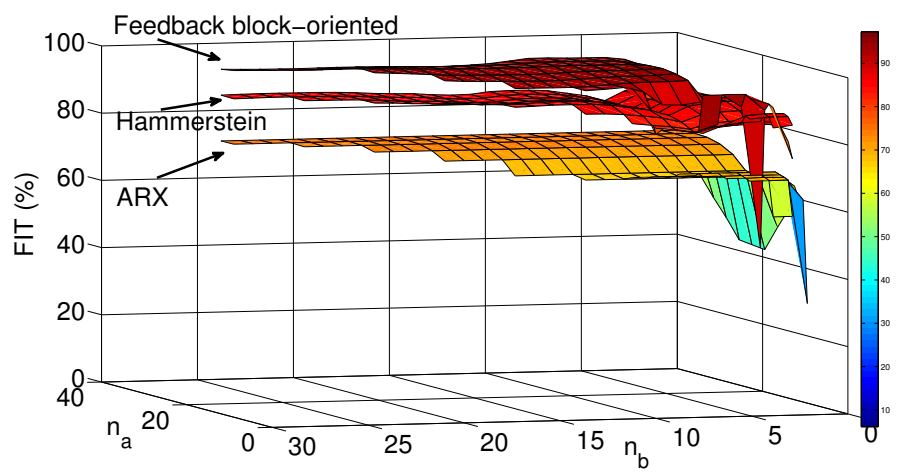

Figure 12: The model fit percentages for varying model order of the linear dynamic block for the ARX, Hammerstein and Feedback block-oriented models

\section{CONCLUSION}

An ARX model is shown as a simple but effective possible linear hydrodynamic model, offering the advantage of a convex optimisation for parameters that can be easily solved. Nonlinear dynamics are investigated by including a nonlinear static block, either in series or in a feedback loop with the linear ARX model. By consideration of the physical meaning of the input/output relationship, the nonlinear static block should model the relationship between the hydrostatic restoring force and the body position. The nonlinear static block is parameterised using a linear combination of basis functions, which can then also be easily identified from NWT experiments designed to produce a matrix of hydrostatic force versus body displacement values.

\section{ACKNOWLEDGEMENTS}

The authors would like to acknowledge the financial support of Enterprise Ireland and the European Regional Development Fund under contract EI/CF/2011/1320. They would like to thank: Tom Kelly and Andrej Roessling for their help in obtaining the BEM hydrodynamic parameters; Tue Trong Vu for his insightful discussions on system identification; the three interns, Marie Cathelain, Louise Guillemet and Thibault Le Heuc for their contributions towards developing the OpenFOAM NWT; the attendees of the CFD for Ocean Energy seminar days at COER for their feedback and advice, especially CADFEM Ireland.

\section{REFERENCES}

Afshar, M. A. (2010). Numerical wave generation in open foam. Master's thesis, Chalmers University of Technology.

Armesto, J. A., R. Guanche, A. Iturrioz, C. Vidal, \& I. J. Losada (2014). Identification of state-space coefficients for oscillating water columns using temporal series. Ocean Engineering 79, 43-49.

Babarit, A. \& P. Laporte-Weywada (2009). On the numerical modelling of the nonlinear behaviour of a wave energy converter. Proceedings of OMAE2009.
Bai, E.-W. (2003). Decoupling the linear and nonlinear parts in hammerstein model identification. Automatica 40, 671-676.

Bhinder, M., A. Babarit, L. Gentaz, \& P. Ferrant (2011). Assessment of viscous damping via $3 \mathrm{~d}$-cfd modelling of a floating wave energy device. In EWTEC 2011.

Bonfiglio, L., S. Brizzolara, \& C. Chryssostomidis (2011). Added mass and damping of oscillating bodies: a fully viscous numerical approach. Recent Advances in Fluid Mechanics, Heat \& Mass Transfer and Biology.

Cathelain, M. (2013). Modelling of a floating object in heave motion using a numerical wave tank in openfoam. Master's thesis, Ecole Centrale de Nantes.

Cummins, W. (1962). The impulse response function and ship motions. Technical report, DTIC Document.

Davidson, J., S. Giorgi, \& J. V. Ringwood (2013). Linear parametric hydrodynamic models based on numerical wave tank experiments. In EWTEC 2013.

Gilloteaux, J.-C., G. Bacelli, \& J. V. Ringwood (2008). A nonlinear potential model to predict large-amplitudes-motions: application to a multi-body wave energy converter. In Proc. 10th World Renewable Energy Conf.

Guérinel, M., M. Alves, \& A. Sarmento (2011). Nonlinear modelling of the dynamics of a free floating body. EWTEC, Southampton.

Higuera, P., J. L. Lara, \& I. J. Losada (2013). Simulating coastal

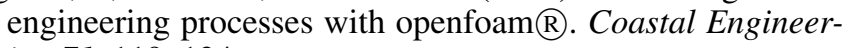
ing 71, 119-134.

Iturrioz, A., R. Guanche, J. A. Armesto, C. Vidal, \& I. J. Losada (2013). Experimental and numiercal development of a floating multi-chamber owc device. In EWTEC 2013.

Jacobsen, N. G., D. R. Fuhrman, \& J. Fredsøe (2012). A wave generation toolbox for the open-source cfd library: Openfoam $囚$. International Journal for Numerical Methods in Fluids 70, 1073-1088.

Lambert, R. J. (2012). Development of a numerical wave tank using openfoam. Master's thesis, Universidade De Coimbra.

Ljung, L. (1999). System identification. Wiley Online Library.

Nelles, O. (2001). Nonlinear System Identification: From Classical Approaches to Neural Networks and Fuzzy Models. Springer.

Palm, J., C. Eskilsson, G. Moura Paredes, \& L. Bergdahl (2013). $\mathrm{Cfd}$ simulation of a moored floating wave energy converter. In EWTEC 2013.

Pearson, R. K. \& M. Pottmann (2000). Gray-box identification of block-oriented nonlinear models. Journal of Process Control 10, 301315.

Perez, T. \& T. I. Fossen (2009). A matlab toolbox for parametric identification of radiation-force models of ships and offshore structures. Modeling, Identification and Control 30, 1-15.

Schmitt, P., S. Bourdier, T. Whittaker, D. Sarkar, E. Renzi, F. Dias, K. Doherty, J. van't Hoff, et al. (2012). Hydrodynamic loading on a bottom hinged oscillating wave surge converter. In ISOPE 2012.

Taghipour, R., T. Perez, \& T. Moan (2008). Hybrid frequencytime domain models for dynamic response analysis of marine structures. Ocean Engineering 35, 685-705.

Tanizawa, K. (2000). The state of the art on numerical wave tank. In Proceeding of 4th Osaka Colloquium on Seakeeping Performace of Ships.

Vyzikas, T., E. Ransley, M. Hann, D. Magagna, D. Greaves, D. Simmonds, V. Magar, \& D. Conley (2013). Integrated numerical modelling system for extreme wave events at the wave hub site. In ICE Conference on Coasts, Marein Structures and Breakwaters.

Zurkinden, A. S., F. Ferri, S. Beatty, J. P. Kofoed, \& M. Kramer (2014). Non-linear numerical modeling and experimental testing of a point absorber wave energy converter. Ocean Engineering 78, 11-21. 